\title{
How Intraoral Pressure Shapes the Voicing Contrast in American English and German
}

\author{
Blake Rodgers \\ University Wisconsin-Madison
}

Susanne Fuchs

Zentrum für Allgemeine Sprachwissenschaft, Berlin

This study examines intraoral pressure for English and German stops in bilabial and alveolar place of articulation. Our subjects are two speakers of American English and three speakers of German. VOICING is the main phonological contrast under evaluation in both word initial and word final position. For initial stops, a few of the pressure characteristics showed differences between English and German, but on the whole the results point to similar production strategies at both places of articulation in the two different languages. Analysis of the pressure trajectory differences between VOICING categories in initial position raises questions about articulatory differences. In the initial closing gesture, time from start of gesture to closure is roughly equivalent for both categories, but the pressure change is significantly smaller on average for VOICED stops. Final stops, however, present a more complicated picture. German final stops are neutralized to a presumed VOICELESS phonological state. English final $/ \mathrm{p} /$ is broadly similar to German /p/, but English /t/ often shows no pressure increase at all which is at odds with the conventional account of phonation termination via pressure increase and loss of pressure differential. The results raise the question of whether the German final stops should be considered VOICELESS or some intermediate form, at least as compared to English final stops.

\section{Introduction}

The aims of this study are to investigate the phonetic realization of the phonological voicing contrast in word initial and final position in American English and German by means of temporal acoustic measures in combination with intraoral pressure. A secondary aim is to develop a set of measures to enable automated measurement of pressure characteristics. 
In American English and German, both VOICED ${ }^{1}$ and VOICELESS stops may be realized without phonation during closure in word initial position (for German: Jessen 1998, Fuchs 2005). For word final position, German is particularly interesting as it is the prototypical language for final neutralization of stop VOICING in coda position (Brockhaus 1995), while English maintains a clear acoustic distinction in this position.

Intraoral pressure is a crucial parameter for phonation because it results from both laryngeal and supralaryngeal mechanisms which are difficult to study in combination. Therefore, the focus here will be on intraoral pressure changes and temporal acoustic measures to characterize word initial and word final stops in these two languages.

Moreover, word position in the sentence will be evaluated by comparing tokens in sentence internal position with tokens in sentence final position. We expect that sentence position could be a crucial factor influencing voicing contrast. First, glottalization or glottal stops are often found at the end of a sentence (Kohler 2001). Second, subglottal pressure, and consequently intraoral pressure, may be reduced due to the air consumption at the end of a sentence. This should in turn strongly influence the transglottal pressure difference and hence, phonation patterns.

In the following sections, we will first describe the phonetic realization of the voicing contrast in American English and German in more detail. Second, we will provide a brief overview of the literature on final devoicing. Third, we will focus on the role of intraoral and subglottal pressure with respect to phonation and finally, we will present the working hypotheses of the current study which are based on the previously reported results from the literature.

\subsection{Phonetic realization of the voicing contrast in American English}

Kent and Moll (1969) noted that the difference between VOICED and VOICELESS categories for stops was due to volume changes and the timing of the glottal opening relative to the upper vocal tract gestures. Specifically, they found that for VOICED stops, vocal tract volume was higher due to expanded pharyngeal walls and lowering of both the hyoid bone and the larynx. Labial and lingual motion for bilabial and apical stops was found to be similar for both categories.

Westbury (1983) observed a collection of articulatory differences between VOICED and VOICELESS stops in American English. VOICED stops were

We used uppercase following Docherty (1992) to indicate phonological contrast and not actual presence or absence of phonation. 'VOICING' is used to designate the abstract phonological category. 
generally characterized by an increase in supralaryngeal volume, and VOICELESS stops occasionally had a decrease in volume. The larynx was always in a lower position for VOICED relative to VOICELESS stops. The tongue root was more advanced for VOICED stops with the exception of utterance initial /b/.

Perkell (1969) found that the larynx rose for /t/ as compared to /d/, which is consistent with the volume observations by Westbury (1983). The pharynx began more constricted for $/ \mathrm{d} /$ and then expanded to a greater volume as compared to $/ \mathrm{t} /$ during closure.

These studies taken together indicate that the volume-expanding gesture seems to be an integral part of VOICED stops in English. This gesture will give rise to at least two of the acoustic parameters that signal VOICING in English, namely voicing during closure and how rapidly phonation is initiated or terminated. The studies also indicate that the expansion gesture can be accomplished with several different strategies.

\subsection{Phonetic realization of the voicing contrast in German}

In his comprehensive acoustic study on the realization of the voicing contrast in German, Jessen (1998) described a series of peculiarities related to the occurrence of the obstruent in a given word (Note that Jessen called the phonological voicing contrast a tense-lax distinction which would be primarily based on durational differences). In his study he considered three positions and found that the stops $/ \mathrm{b} \mathrm{d} \mathrm{g/} \mathrm{and} / \mathrm{p} \mathrm{t} \mathrm{k/} \mathrm{can} \mathrm{be} \mathrm{distinguished} \mathrm{primarily} \mathrm{by}$ aspiration duration rather than voicing in utterance initial stressed position (\#\#_V) and also in the post-voiceless position (\#_V). The phonologically voiced stops / b d g/ are often devoiced in these positions. Devoiced is a phonetic term which describes a token that is realized without significant voicing during oral closure. Different degrees of devoicing can occur. The stops $/ \mathrm{b} \mathrm{d} \mathrm{g/} \mathrm{and} \mathrm{/p} \mathrm{t} \mathrm{k/}$ can be distinguished by voicing during closure and by aspiration duration in intervocalic V_V position. In Fuchs (2005) the articulatory realization of the voicing contrast was investigated in word initial, word internal and word final position. Her data provided evidence that glottal abduction was mainly a property of VOICELESS stops in word initial position. In all other positions, supralaryngeal correlates and temporal differences played the major role.

To summarize, the production of the voicing contrast in German varies consistently with the position of the obstruent in the word. Voicing contrast may be a somewhat misleading term, since in word initial position the contrast is primarily based on aspiration. Voicing during closure may occur rarely. 


\subsection{Final devoicing}

Final obstruent devoicing or so called "Auslautverhärtung" in German has been a major issue in phonological debates and one of "most popular of German phonological rules" (Giegerich, 1989, p.51). The phonological rule itself refers to the loss of voicing for VOICED obstruents in word, morpheme or syllable final position, for example:

$\begin{array}{ll}\text { Orthography and English translation } & \text { Phonetic transcription } \\ \text { Rad (wheel) vs. Rat (advice) } & \text { [Ra:d] vs. [Ra:t] } \\ \text { lies (read!) vs. ließ (let) } & \text { [li:z] vs. [li:s] }\end{array}$

Historically it seems to have emerged in the transition between Old High German and Middle High German.

Final devoicing has been explained as feature changing in the Sound Patterns of English (SPE) tradition, reduction or spreading (Mascaró 1987) and licensing (Lombardi 1991, 1995, 1999) (for a comprehensive overview, see Brockhaus 1995). From a phonetic standpoint final obstruent devoicing is related to the question of whether VOICED stops are produced in similar fashion to their VOICELESS counterparts (full neutralization) or some differences can be observed (partial neutralization). The question arises as to how much phonetic detail should be taken into account to speak for one or the other. Several phoneticians investigated the issue of neutralization by means of acoustic data. Evidence was provided in both directions - for full (e.g. Fourakis and Iverson 1984) and for partial neutralization (e.g. Port and Crawford 1989, Port and O'Dell 1985), and the debate became more and more centered on methodological issues. Results were dependent on statistical methods, speech corpora (read versus natural speech, isolated words versus words in a frame sentence, nonsense words versus real words, frequently occurring words versus not frequently occurring words) and experimental set-ups (the subjects were/were not aware of the research question, hyperarticulated versus more natural speech conditions etc.). Regional variations could also be found, e.g. South German speakers clearly maintain the contrast (Piroth \& Janker 2004).

It is challenging to get a comprehensive picture of all articulatory motions involved in the voicing contrast. A more indirect measure directly driving the phonation patterns may be to investigate intraoral pressure, since it is the consequence of the orchestra of articulatory motions. 


\subsection{Intraoral pressure and the voicing contrast}

Measuring changes in intraoral pressure are of particular advantage to investigate the voicing contrast, since they directly mirror the combination of the manifold articulatory actions at the laryngeal and supralaryngeal levels. For instance, intraoral pressure will automatically increase when the vocal tract is closed by the lips and the velar port. Moreover, it will increase rapidly up to the level of subglottal pressure (ceiling effect) when the glottis is open and there is continuous air supply from the lungs. In contrast, intraoral pressure may be relatively low when the glottis is closed or the supralaryngeal articulators are moved in such a way that the oral cavity is enlarged (so called cavity enlargement). Various articulatory actions are reported in the literature (for a review, see Westbury 1983, Fuchs 2005) which describe the enlargement of the oral cavity leading to the same acoustic result - the maintenance of voicing. These processes are known under the term 'motor equivalence'. They are often speaker- or even phoneme-specific and can make an investigation of the voicing contrast difficult. The orchestra of articulatory maneuvers is directly reflected in the changing intraoral pressure and the respective phonation. Phonation is primarily driven by the transglottal pressure difference (difference between subglottal and intraoral pressure) and the vocal fold tension. There are few studies investigating intraoral pressure measures with respect to the voicing contrast.

Müller and Brown (1980) investigated intraoral pressure and the voicing contrast in American English and developed a measure of the concavity of the intraoral pressure increase that compares initial slope of the pressure increase to final slope. [alpha] is the initial slope and [beta] is the final slope. The measure [delta] is defined as [alpha] - [beta]. [delta] greater than zero implies a convex shape, while [delta] less than zero implies a concave shape. The authors found that VOICELESS stops had higher values of [delta] than VOICED ones. They also found, however, that in many cases, it was difficult to define this parameter due to irregularly shaped pressure curves.

Müller and Brown (1980) built on the vocal tract pressure model developed by Rothenberg (1968), and concluded that the only way to achieve a convex pressure trajectory was to include an active volume expansion in the model. Koenig and Lucero (2008) observed that intraoral pressure can provide insights into such expansion gestures, if present, and more broadly provide insights into pressure control for the purpose of maintaining voicing during stop closure. 


\subsection{Subglottal pressure and the voicing contrast}

Since air is consumed during speech production, subglottal pressure typically decreases over longer periods of speech. A lower subglottal pressure at the end of a sentence in comparison to the beginning or middle of a long sentence should also affect the upper limit of the intraoral pressure in obstruent production, since intraoral pressure equalizes subglottal pressure when the glottis is open. Moreover, the transglottal pressure difference crucial for phonation may be compromised too, if the intraoral pressure is influenced by vocal tract closure and does not decrease at the same rate as subglottal pressure. Thus, one could suppose that devoicing of VOICED phonemes may occur even more frequently or more rapidly in sentence final position due to the lower subglottal pressure. Interactions with other mechanisms, like the decrease of vocal fold tension in sentence final position, may be plausible as well, and will influence voicing during closure.

\subsection{Assumptions and predictions}

1. Since previous studies of American English and German reported no differences with respect to phonation in word initial stops, we predict that the pressure increase (slope) and pressure peak will behave similarly for VOICED and VOICELESS stops and for German and American English in syllable initial position.

2. If German word final stops are, in fact, neutralized, we assume no statistical difference between the two voicing categories. However, from a phonetic point of view, it may still be possible that some minor residue of the voicing contrast remains which could speak for a partial neutralization from a speech production point of view.

3. For American English we expect the maintenance of the voicing distinction in word final position with a shallower intraoral pressure rise and lower pressure peak for the VOICED stops and a steeper pressure rise and higher pressure peak for the VOICELESS ones. In addition, we suppose that if German final stops neutralize, they will resemble the English final VOICELESS stops.

4. We expect that sentence position will influence the realization of the voicing contrast. 


\section{Methodology}

\subsection{Instrumentation}

To record intraoral pressure (IOP), an experimental setup was designed whereby a piezoresistive ${ }^{2}$ pressure transducer Endevco 8507C-2 measuring about $2.4 \mathrm{~mm}$ in diameter and $12 \mathrm{~mm}$ in length was affixed to the rearmost portion the hard palate. The sensor measures the difference between intraoral and atmospheric pressure, the latter being obtained via a tube passed through the teeth. This arrangement offers several advantages: It permits simultaneous recording of acoustics and intraoral pressure, it is not affected by saliva blocking the tube, it is more comfortable for speakers than inserting a tube or catheter through the nose, and it allows recording of a high quality acoustic signal in comparison to a Rothenberg mask. Data was collected for bilabial, alveolar and velar stops. Data for velar stops was found to be unreliable due to the placement of the sensor in the vicinity of velar occlusion. Pressure can rapidly drop to zero if occlusion occurs aft of the sensor, so velar data was not included in the analysis.

\subsection{Speakers and speech material}

All together four German and four American English speakers (two males and two females per language) were recorded at the phonetics laboratory at ZAS, but only 3 American speakers and 2 Germans have been analysed so far. All German speakers are from the northern parts of Germany and Berlin. The American English native speakers are from different areas in the US (California, Missouri, Delaware).

Table 1: Speech material of American English target words

\begin{tabular}{|c|c|c|c|c|c|}
\hline & \multicolumn{5}{|c|}{ Word initial (onset) / Word final (coda) } \\
\hline & $\mathbf{a}$ & $\mathbf{u}$ & i & ae & $\Lambda$ \\
\hline $\mathbf{p}$ & pop & poop & peat / deep & pack / tap & pup \\
\hline b & bog / gob & boot / tube & bead / hebe & bat / cab & butt / tub \\
\hline $\mathbf{t}$ & top / cot & tube / boot & teak / peat & tap / bat & tub / butt \\
\hline d & dock / god & dude & deed & dad & duck / cud \\
\hline
\end{tabular}

Monosyllabic target words were selected to investigate the voicing contrast in onset and coda position of American English (see Table 1). In one case no real

2 This device changes its electrical resistance in response to changing mechanical pressure, which allows measurement of a voltage output that is proportional to intraoral pressure. 
word existed in the lexicon, so a phonotactically comparable word was created ('hebe').

Moreover, the VOICED and VOICELESS stops were followed by different vowel contexts, but these will not be investigated at this point. All target words (X) occurred two times in a frame sentence, once in sentence medial position and once at the end of the sentence ("Get THIS new X for me, no, get THAT new X."). Sentence focus was placed words other than the target words in order to avoid an enhancement of the voicing contrast due to emphasis. The emphasis was placed on the two words written in capital letters (THIS and THAT).

For the German speech material we tried to make the corpus as similar as possible to the English characteristics. However, monosyllabic words are not as frequent in German as in American English, so it was sometimes difficult to find real words. Three vowels were chosen, both tense and lax, for a total of six vowels. The target words are listed in Table 2 . All target words were embedded in the frame sentence: "Ich meine ZWEI X am Morgen, nicht DREI X". (I mean TWO $X$ in the morning, not THREE $X$ ) with the accent on TWO and THREE. We did not find any combinations of lax vowels ending with a voiced stop, except from Klub (which is a loan word from English club).

Table 2: Speech material of German target words (\# = gap in this particular combination)

\begin{tabular}{|l|l|l|l|l|l|l|}
\hline & \multicolumn{5}{|c|}{ Word initial (onset) / Word final (coda) } & \\
\hline & a & _u & i & A & U & I I \\
\hline $\mathbf{p}$ & Paar/Paap & Pup & Piek/Piep & Pack/Kapp & Putz/Stupp & Pils/Kipp \\
\hline $\mathbf{b}$ & Bad/Stab & Bub & Biest/Dieb & Bass/\# & Bus/Klub & Biß \\
\hline t & Tag/Tat & Tuch/Gut & Tief/Reif & Taft/Patt & Tusch/Schutt & Tisch/Pitt \\
\hline d & Dart/Bad & Dur/Sud & Dieb/Lied & Dach/\# & Duft/\# & Ding \\
\hline
\end{tabular}

\subsection{Labeling procedures and further calculations}

The acoustic signal had a sampling frequency of $44 \mathrm{kHz}$ and the pressure signal is sampled at $1375 \mathrm{~Hz}$. The pressure signal is first low-pass filtered using a 61 tap FIR filter with a passband of $6.875 \mathrm{~Hz}$. The filtered signal is used for calculating the derivatives and the slope of the pressure rise. The first and second derivative of this signal is determined, and the second derivative is filtered with the same low-pass filter. First derivative of pressure signal will be referred to as pressure velocity, and second derivative of pressure signal will be referred to as pressure acceleration.

Start and end of pressure excursion were determined by peaks in the pressure acceleration signal. For tokens that did not present a well defined peak, 
the start was marked as the first upward spike in the pressure acceleration, and similarly, the end was marked as the last upward spike. The markings in these cases were confirmed by inspection of the smoothed pressure curve and subjective determination of beginning or end of pressure excursion.

Time of closure was estimated using the zero crossing of the pressure acceleration. Müller and Brown (1980) marked closure as the time when oral airflow dropped to zero. They also observed that the closure event generally causes a change in the sign of the pressure acceleration from positive to negative. As we did not measure airflow in the present study, the second method will be used to estimate closure time. A small subset of VOICED tokens did not show a sign change, and closure time was estimated by visual inspection.

TF32 (Milenkovic 2005) was used for determination of voicing offset, voicing onset and release. For voicing onset and voicing offset, a high-pass filtered pressure signal was used in conjunction with the spectrogram to determine where voicing was present. Voicing was defined as presence of a coherent oscillation in the pressure signal. This method proved to be more sensitive than phonation data from either the acoustic plot or the spectrogram. In other words, pressure ripples were detected beyond either the acoustic ripples or the voicing bar. Release was marked as onset of frication noise when present, and subjective determination of a pressure knee or sudden drop in the pressure curve when frication noise was not present.

The pressure knee was defined as the peak for VOICED tokens and the end of the initial rise for VOICELESS tokens. The knee was defined in this way to enable a determination of the initial slope of the pressure curve. VOICED tokens typically (though not always) showed a steady rise in pressure until release. VOICELESS tokens typically showed a rapid rise to the voicing offset point (Figure 1).

Time points defined for measurement are (1) start of pressure excursion (t0), marked as peak in smoothed pressure acceleration curve, (2) closure (tclos), which was approximated by the point where the pressure acceleration curve changed sign (following Müller and Brown, 1980), (3) voicing offset and (4) release (trel), which was determined from the acoustic burst. The time point of the knee (tknee) was defined as the offset of voicing for VOICELESS stops and release time for VOICED stops. The knee measurement is intended to capture the first major break in the smoothed pressure trajectory. Pressure at knee relative to pressure at closure allows a rough determination of the overall initial pressure slope. 

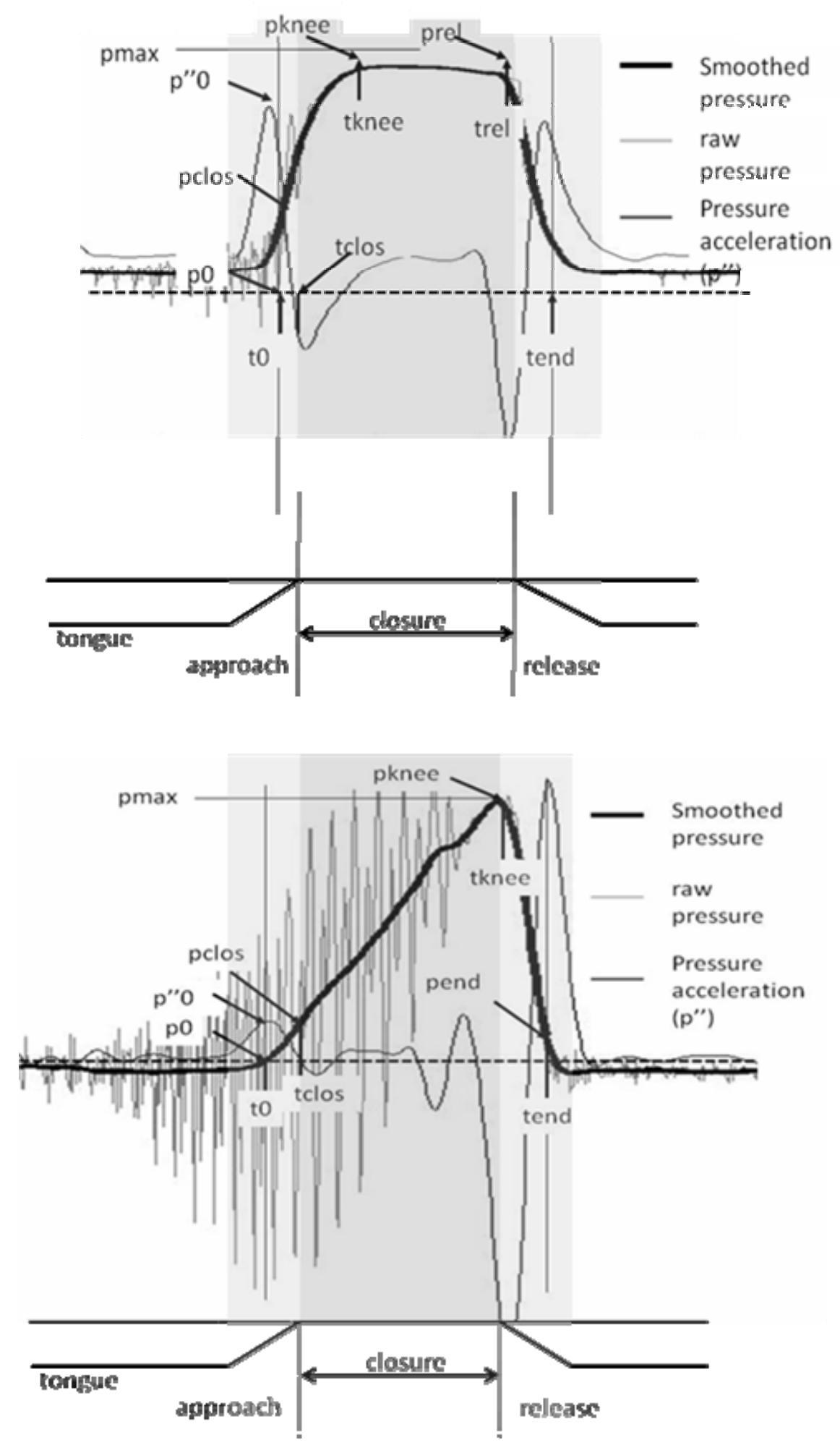

Figure 1. Pressure plots for word initial position a) VOICELESS stop and b) VOICED stop. Raw pressure signal is gray, and smoothed pressure signal is the heavy black curve.

\subsection{Statistical design}

The open software package R (R 2010) was used for statistical computing. Since voicing contrast is the major topic we are interested in and speakers may behave 
differently, we first split all the data according to speaker, position of the consonant in the word, place of articulation and sentence position and ran several t-tests with a temporal or pressure parameter as the dependent variable and voicing contrast as the independent factor. We considered all differences with $\mathrm{p} \leq 0.05$ as significant, but additionally accounted for multiple comparisons by applying a Bonferroni correction ( $0.05 /$ number of tests run).

As a dependent variable we selected one of the temporal or pressure parameters, as fixed effects we chose voicing contrast, language, and sentence position and as random effects speaker and place of articulation. Random effects also have an influence on the variance in the data, but these effects are eliminated in the analysis. Data for word initial position will be presented first in the results section, followed by data for coda position.

\section{$3 \quad$ Results}

\subsection{Initial stops}

German and English stops in syllable initial position were found to be qualitatively similar, but there were significant differences in several pressure measures that will be discussed later. Initial VOICELESS stops are characterized by four phases. The first phase begins with a pressure increase. This is brought about by initiation of the tongue gesture possibly influenced by the glottal opening. Müller \& Brown (1980:358) found in their modeling that the size of the glottal opening in the initial phase of VOICELESS stops influenced the slope of the initial pressure rise. Curvature of the pressure trajectory is positive until closure, which is the start of the second phase. At this point curvature becomes negative. Pressure velocity typically increases for the first portion of the pressure rise, and then decreases until phonation ceases. The third phase has no phonation. Pressure is relatively flat, but can increase or decrease slightly, typically at a steady rate. The fourth and final stage begins at release of occlusion. The pressure drops back to ambient, and phonation typically resumes as pressure nears ambient for VOICELESS stops. Figure 2 shows average pressure plots for four speakers. 
English

BR

a)

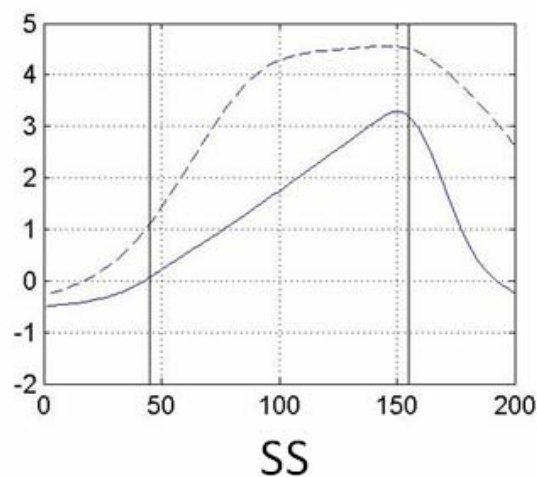

c)

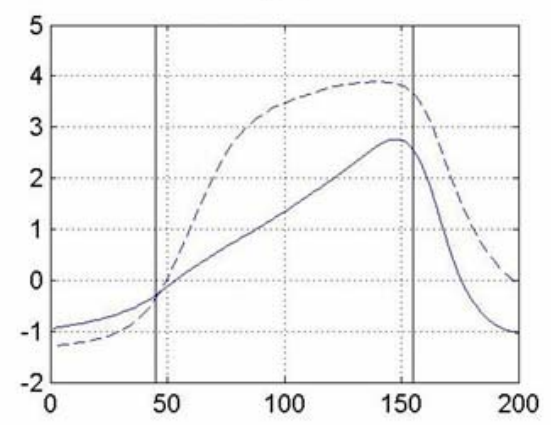

German

JD
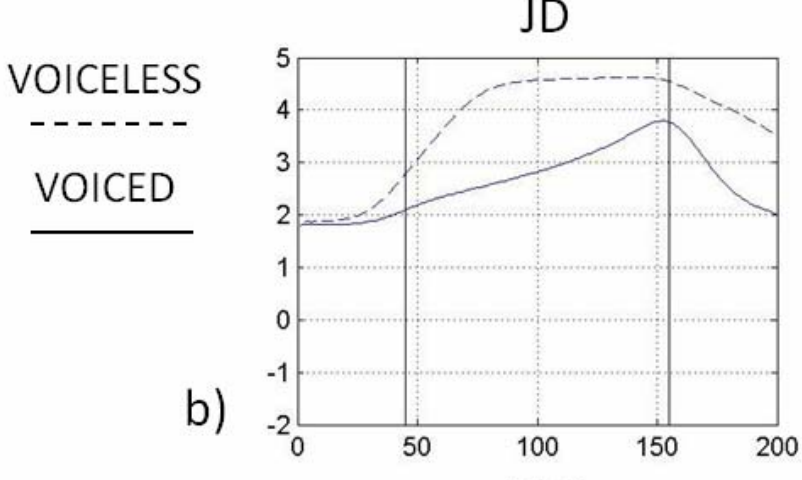

RW

d)

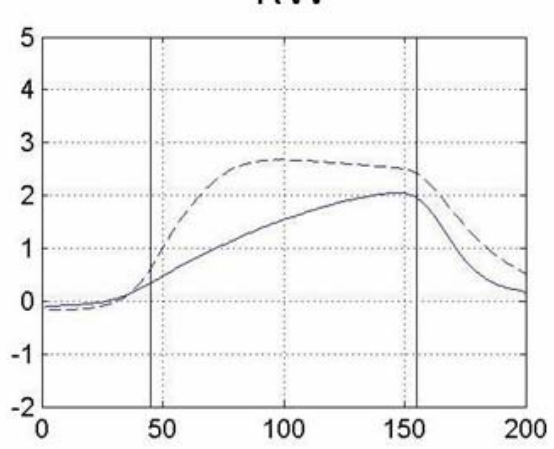

Figure 2: Average pressure plots for initial alveolar stops for individual speakers. Time alignment is at closure time and release time.

Speaker RW showed a consistently concave pressure plot for VOICED tokens, with the exception of a few samples. This can also be seen in his average plots, which are more concave for VOICED stops than those of the other speakers, English or German. This might suggest a weaker volume expansion gesture.

Initial VOICED stops are characterized by three identifiable phases. Like VOICELESS stops, they begin with the start of the tongue trajectory toward occlusion, and move to the second phase at occlusion. VOICED stops typically have no phase corresponding to the third phase of VOICELESS stops, instead having a second phase characterized by rising pressure until the third stage is initiated at release. The rate of pressure increase is smaller than that for VOICELESS stops, and the rate of pressure change is typically flat or decreasing. Phonation typically continues until release, at which point pressure falls back to ambient. In some rare cases, phonation stops for a brief interval prior to release. 
How Intraoral Pressure Shapes the Voicing Contrast in American English and German

Alveolar stops

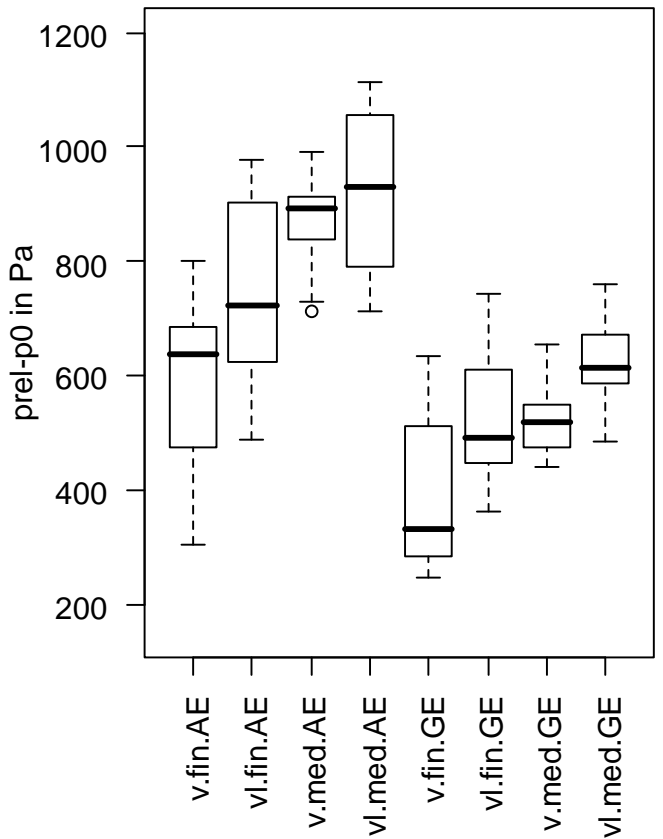

Bilabial stops

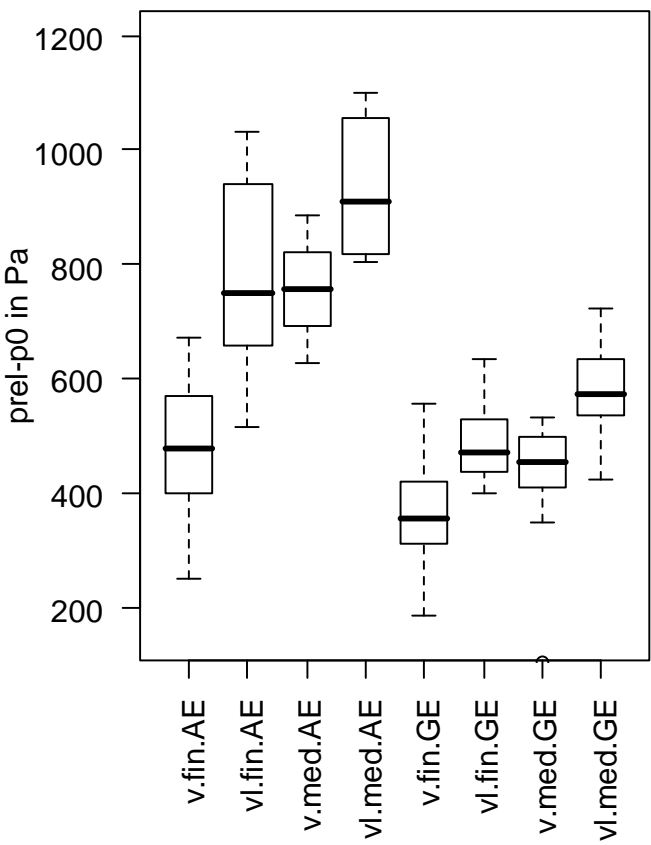

Figure 3: Release pressure relative to initial pressure.

Figure 3 shows the release pressure as compared to initial pressure. English speakers show consistently higher pressures for comparable stops. Figure 4 shows the maximum pressure acceleration. Interestingly, German speakers show higher values for this parameter, which is the opposite of what would be expected from the maximum pressure comparison.

Alveolar stops

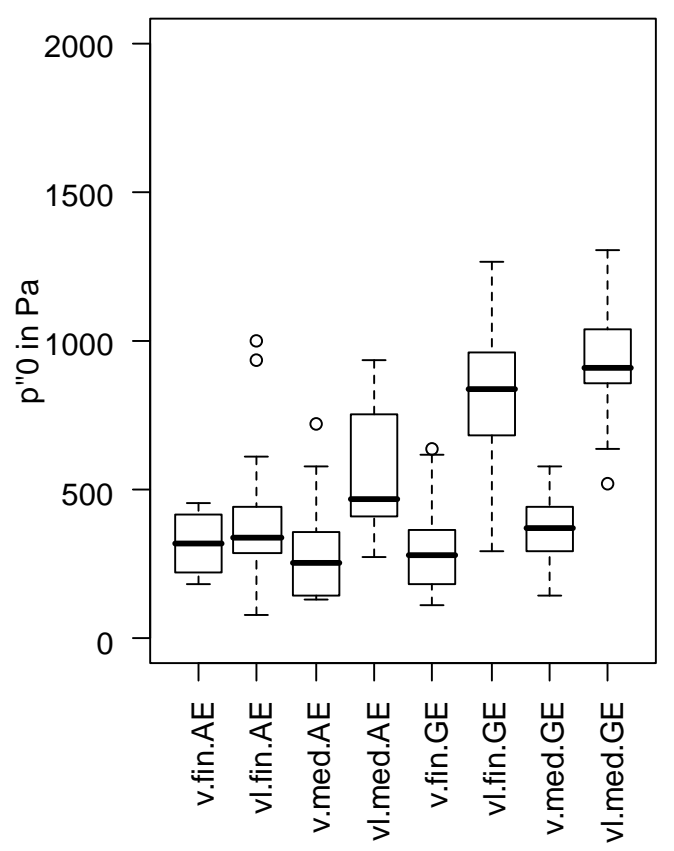

Bilabial stops

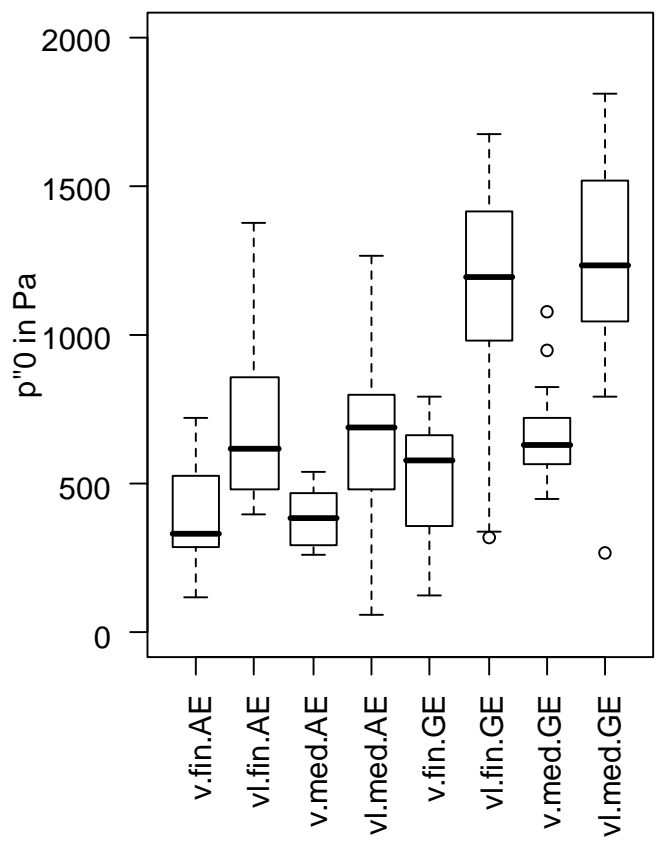

Figure 4: Pressure acceleration peak. 

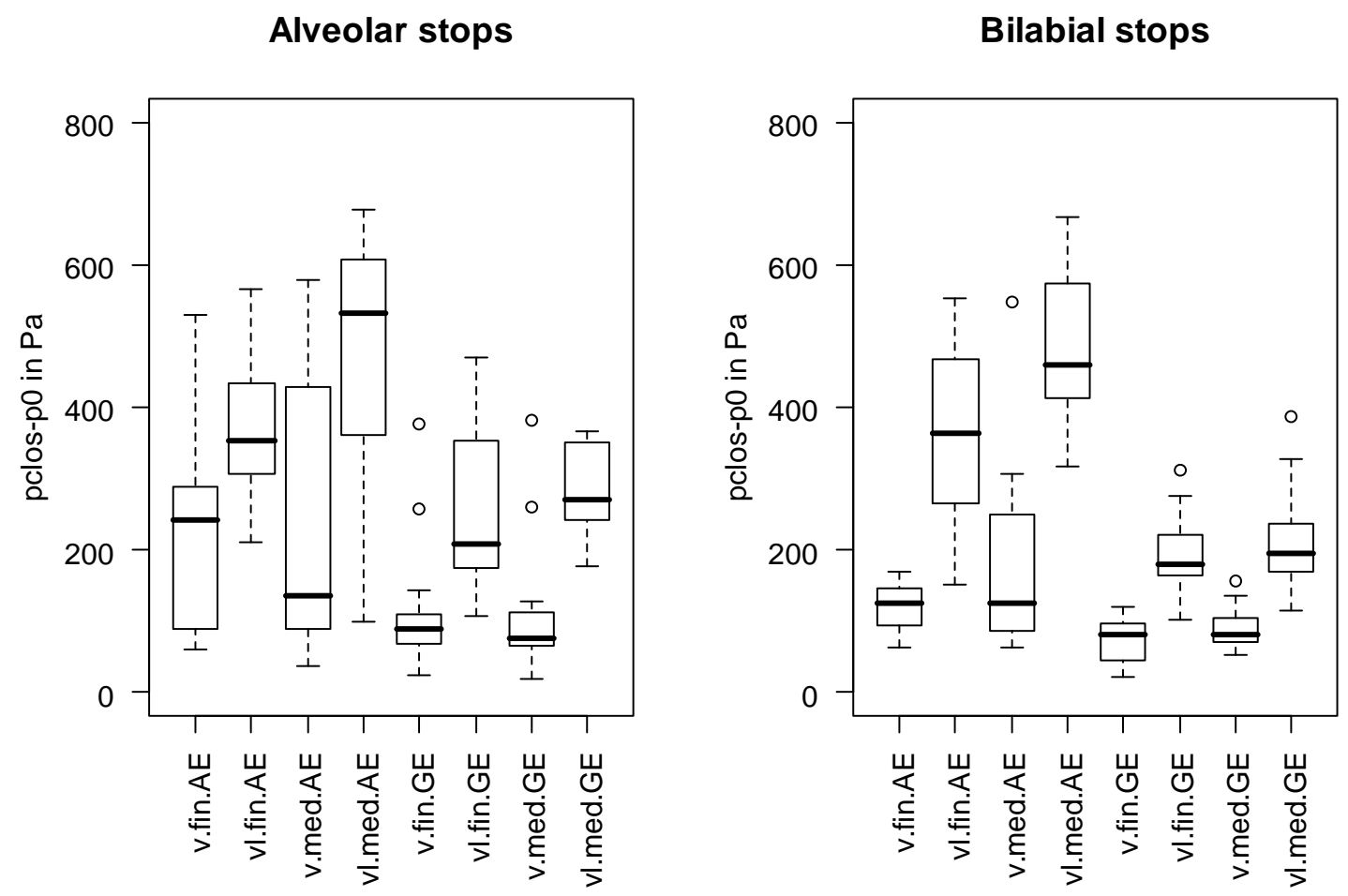

Figure 5: Close pressure relative to initial pressure.

Pressure at closure, as shown in Figure 5, shows that the English speakers are higher for this parameter, which is consistent with the lower pressure acceleration. Figure 6 shows the maximum pressure velocity. There are no clear trends between English and German. It does, however, show a clear difference between the VOICED and VOICELESS categories.

Alveolar stops

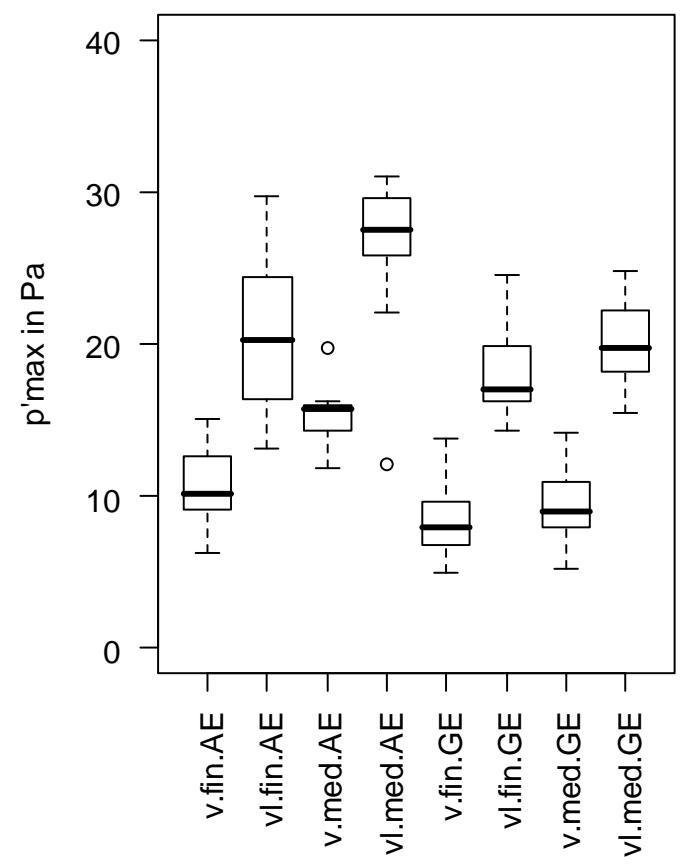

Bilabial stops

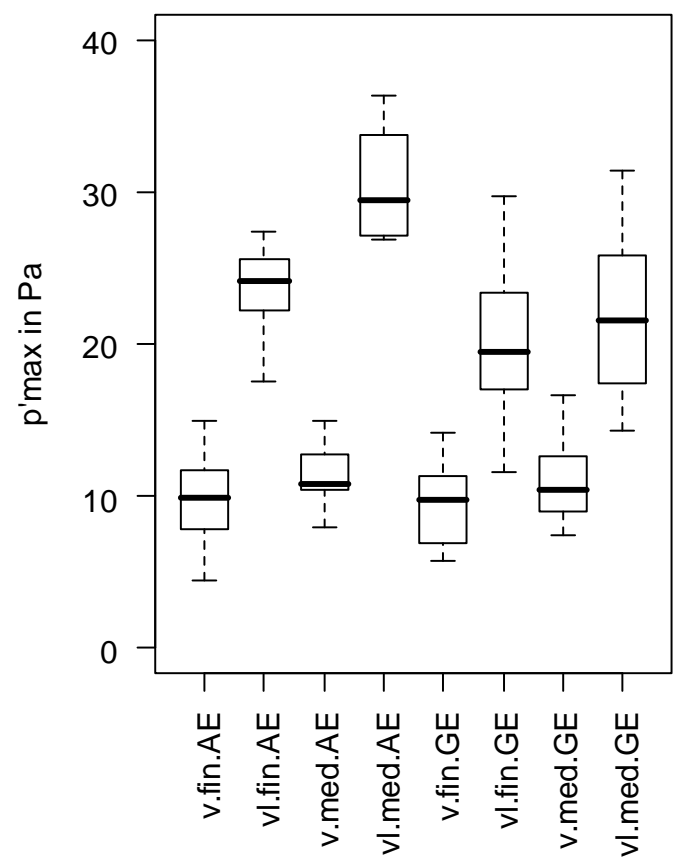

Figure 6: Maximum pressure velocity. 


\subsection{Final stops}

There was a problem with the English carrier phrase for sentence internal final stops. The following fricative and its associated pressure increase completely changed the pressure characteristics. As a result, these tokens were not directly comparable to the other stops in the study, and so were excluded.

English final VOICED stops are characterized by significant voicing into closure and English final VOICELESS stops are characterized by termination of voicing often prior to pressure increase. Some tokens of final VOICELESS stops showed no intraoral pressure rise for the English subjects. Thus, for the latter group, a laryngeal mechanism for termination of voicing other than pressure increase is implicated. For many English final VOICELESS tokens, the voicing terminated prior to any pressure increase at all. This resulted in certain measures being undefined or aberrant for this category. These samples were excluded from analysis. For alveolar place of articulation, phonation terminated with no pressure increase for all tokens for speaker BR, and a majority of tokens for speaker SS. For bilabial place, phonation often terminated with no pressure increase. Speaker SS had 5 out of 8 tokens for final $/ \mathrm{p} /$ that were terminated with no pressure increase. Speaker BR had three tokens that seemed to terminate like the German final /p/, but the other five had much smaller pressure increases, and two of the five didn't actually terminate until after the pressure passed the peak. As a group, the English final /p/ tokens had much smaller pressure at the knee than the German tokens. The conclusion is that the English and German final VOICELESS stops are realized with different articulatory mechanisms, and possibly the two English speakers utilize different strategies as well depending on the adjacent vowel.

English final VOICED stops have pressure curves that are generally flat or convex during the rise, with rare instances of irregular or concave shapes. Pressure acceleration tends to hover around zero with several sign changes (Figure 7a,c).

German final stops are almost completely neutralized to a passive termination of voicing. The pressure rises due to occlusion, and voicing terminates when the pressure differential drops. German final stops, both VOICED and VOICELESS, are characterized by a concave rising pressure slope. This is confirmed by the pressure acceleration, which generally remains negative after closure. Voicing terminates near the peak of the pressure excursion. The statistical differences between VOICING categories are not significant (Figure $7 \mathrm{~b}, \mathrm{~d}$ ). Figure 8 shows the pressure acceleration peak at initiation of stop gesture. As with initial stops, German pressure acceleration values are consistently higher than American English. 
English

a)

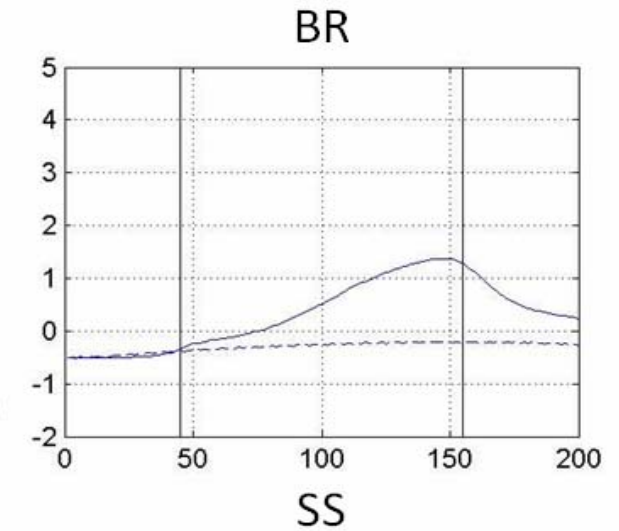

c)

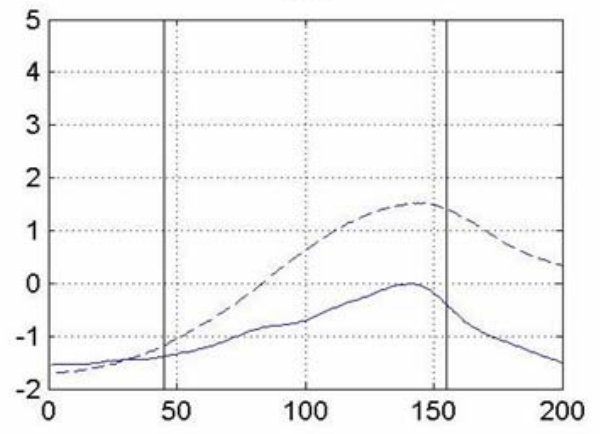

German

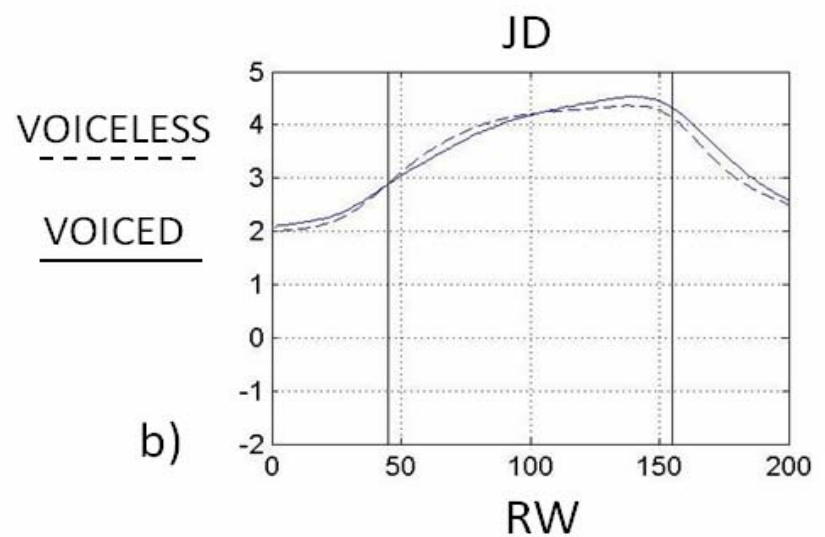

d)

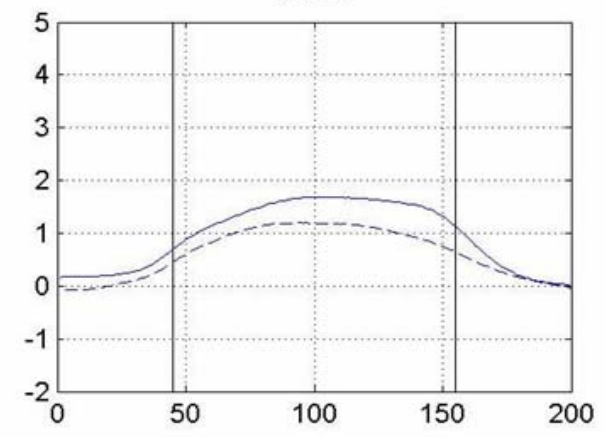

Figure 7: Average pressure for syllable final alveolar stops for individual speakers. Time alignment is at closure time and release time.

Alveolar stops

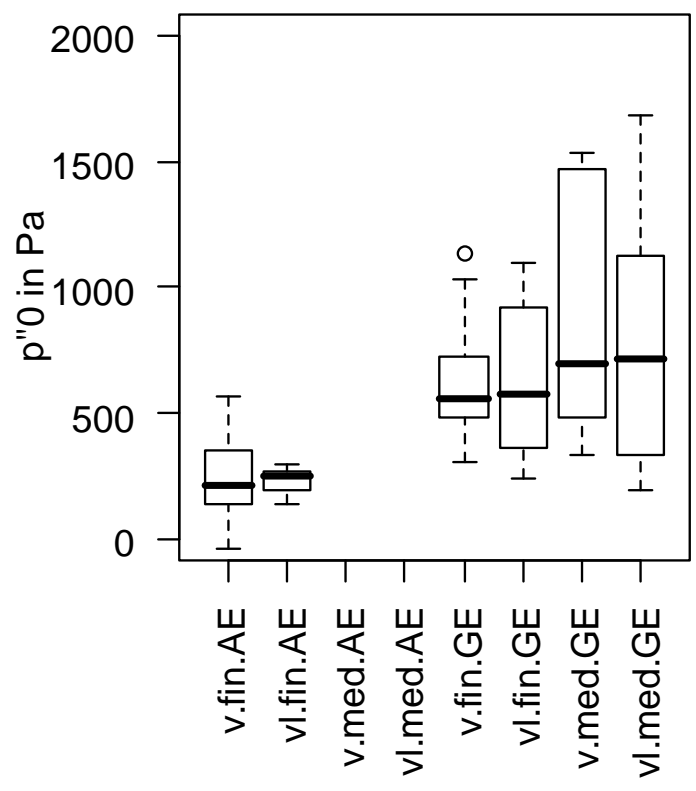

Bilabial stops

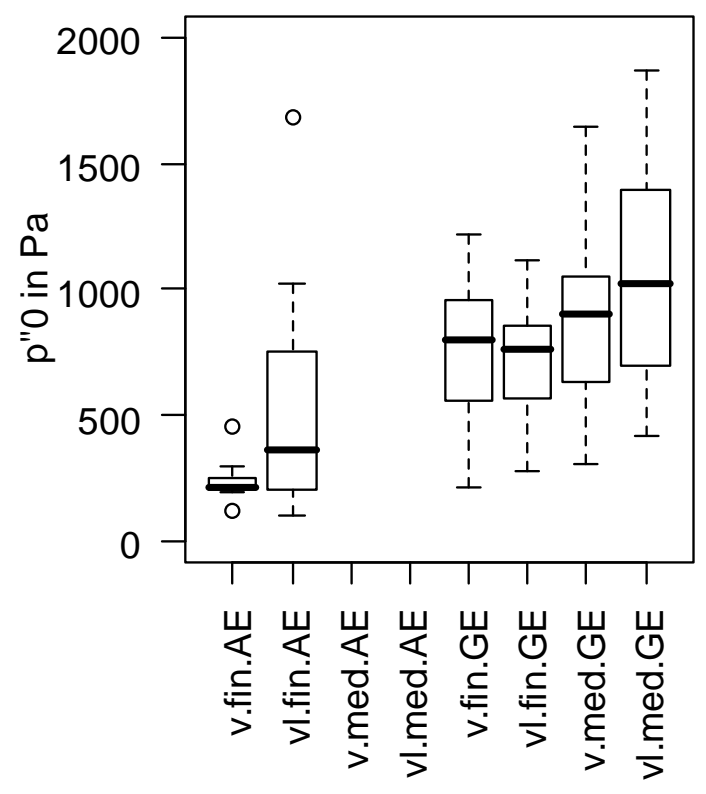

Figure 8: Maximum pressure acceleration for syllable final stops 


\section{Discussion}

Müller and Brown (1980) performed simulations of pressure for intervocalic stops using a model developed by Rothenberg (1968). The model included vocal tract wall impedance, glottal area, supraglottal constriction area and volume of the supraglottal cavity. Running the simulation with fixed wall stiffness, fixed source pressure, laryngeal gestures that changed glottal area and supralaryngeal gestures that changed constriction area produced a pressure trajectory that was always convex (negative pressure acceleration) on the upslope. In order to get a concave or linear pressure trajectory, they found that it was necessary to include an active volume adjustment in the model. As the volume is increased, this will tend to lower the pressure acceleration, and in the return phase of the gesture to original state the pressure acceleration will be higher than otherwise.

Following this line of reasoning, all gestures for which the pressure curve has a linear or concave pressure rise will be assumed to have an associated volume expansion gesture. Absence of these characteristics, however, does not necessarily imply the absence of a volume expansion gesture.

\subsection{Initial stops}

The pressure curves for initial VOICED stops in English are generally flat or slightly concave. For the initial VOICED stops of the two German speakers, one showed a predominantly convex pressure curve, and one showed a predominantly concave pressure curve.

Using the zero crossing of pressure acceleration is presumed to be a reliable marker for closure except in the case of an active volume expansion gesture initiated prior to closure. It is unlikely that the difference in the pressure change from start of gesture to closure between VOICING categories is due to vocal tract wall compliance because the vocal tract is still open through this phase. The difference is presumably due to an active volume expansion during the closure phase, a difference in tongue shape (alveolar only), or a difference in the speed of articulation.

\subsection{Final stops}

Only the English VOICED final stops show a concave pressure curve, and therefore are assumed to have an active volume expansion. The English VOICELESS final stops appear to have two distinct pressure characteristic curves depending on preceding vowel, speaker, and place of articulation. One type shows little or no pressure increase at termination of phonation, and the other shows a small pressure increase similar to initial position. The neutralized 
German final stops show a pressure characteristic that is consistent with passive termination of voicing. These stops are qualitatively different than either class of English final stop.

\section{Conclusion}

The pressure data allows a finer analysis of stops than acoustic data alone. There are broad differences in the small number of speakers considered between English and German. One mystery is the relationship between maximum pressure and maximum pressure acceleration. If the stops were produced in roughly the same manner, an increase of one of these parameters would seem to imply an increase in the other.

The data shows that English maximum pressure is higher, while German pressure acceleration is higher. It's not clear whether this implicates a different production mechanism or simply differences in articulator timing.

The pressure data shows that English final stops fall into at least two distinct categories. It also shows that German final stops, which are mostly neutralized, are qualitatively distinct from either VOICING category of English final stops. This raises the question of the phonological status of the final stops. From a phonetic standpoint, English VOICELESS final stops appear to have a gesture to terminate voicing, while VOICED stops appear to have a gesture to prolong voicing. The neutralized German stops seem to represent stop production with neither of these gestures.

One of the goals of this study was to identify a set of measures that allow automated comparison of pressure curves for stops. Pressure acceleration derived from smoothed pressure data allows marking of several time points including start of gesture (initial peak), closure (sign change), release (negative peak) and end of gesture (final peak). 


\section{References}

Brockhaus, Wiebke (1995). Final Devoicing in the Phonology of German. Tübingen, Germany: Niemeyer.

Docherty, Gerard J. (1992). The Timing of Voicing in British English Obstruents. Berlin: Foris.

Fourakis, Marios and Iverson, Gregory K. (1984) On the incomplete neutralisation of German final obstruents. Phonetica 41: 140-149.

Fuchs, Susanne (2005). Articulatory correlates of the voicing contrast in alveolar obstruent production in German. ZAS papers in Linguistics 41.

Giegerich, Heinz J. (1989). Syllable structure and lexical derivation in German. Bloomington: Indiana University Linguistics Club.

Jessen. Michael (1998). Phonetics and Phonology of Tense and Lax Obstruents in German. (Series: Studies in Functional and Structural Linguistics 44, John Benjamins: Amsterdam.

Kent, Ray D., \& Moll, Kenneth L. (1969). Vocal-tract characteristics of the stop cognates. The Journal of the Acoustical Society of America, 46(6B). 1549-1555.

Koenig, Laura L., \& Lucero, Jorge C. (2008). Stop consonant voicing and intraoral pressure contours in women and children. The Journal of the Acoustical Society of America, 123(2), 1077-1088.

Kohler, Klaus J. (2001). Plosive-related glottalization phenomena in read and spontaneous speech. A stød in German? In Grønnum, N. \& Rischel, J. (eds.), To Honour Eli Fischer-Jørgensen. Festschrift on the Occasion of her 90th Birthday, February 11th, 2001 (Travaux du Cercle Linguistique de Copenhague 31), 174-211. Copenhagen: C. A. Reitzel.

Lombardi, Linda (1991). Laryngeal Features and Laryngeal Neutralization. Ph.D. dissertation, University of Massachusetts, Amherst. Published 1994 by Garland Publishing.

Lombardi, Linda (1995). Laryngeal neutralization and syllable wellformedness, Natural Language and Linguistic Theory 13: 39-74.

Lombardi, Linda (1999). Positional faithfulness and voicing assimilation in Optimality Theory, NLLT 17:276-302.

Mascaró, Joan (1987). A reduction and spreading theory of voicing and other sound effects. Barcelona, Universitat Autònoma. Unpublished manuscript. [Published 1995 in Catalan Working Papers in Linguistics, 4(2). 267-328.]

Milenkovic, Paul (2005). TF32 [Computer software]. Madison, WI: University of WisconsinMadison.

Müller, Eric M., \& Brown, W. S. (1980). Variations in the supraglottal air pressure signal and their articulatory interpretation. In N. J. Lass (Ed.), Speech and language: Advances in basic research and practice, vol. 4, Academic Press.

Perkell, Joseph S. (1969). Physiology of speech production: Results and implications of a quantitative cineradiographic study. Cambridge, MA: The MIT Press. 
Piroth, Hans G. \& Peter M. Janker (2004). Speaker-dependent differences in voicing and devoicing of German obstruents, Journal of Phonetics 32: 81-109.

Port, Robert \& Crawford, Penny (1989). Incomplete neutralization and pragmatics in German, Journal of Phonetics, 17: 257- 282.

Port, Robert F. and O'Dell, Michael L. (1985). Neutralization of syllable-final voicing in German, Journal of Phonetics 13: 455-471.

R Development Core Team (2010). R: A language and environment for statistical computing. Vienna, Austria.

Rothenberg, Martin R. (1968). The Breath-Stream Dynamics of Simple-Released-Plosive Production Vol. 6. Basel: S. Karger.

Westbury, John R. (1983). Enlargement of the supraglottal cavity and its relation to stop consonant voicing. The Journal of the Acoustical Society of America, 73(4), 13221336. 